\title{
Potencialidades e desafios do trabalho da equipe itinerante no cuidado ao usuário de drogas*
}

\author{
Adriane Domingues Eslabão $0^{1,2}$ \\ Leandro Barbosa de Pinho ${ }^{1}$ \\ Márcio Wagner Camatta ${ }^{1}$ \\ Elitiele Ortiz dos Santos ${ }^{1}$ \\ Talita Portela Cassola ${ }^{1}$ \\ Vanessa Alvez Mora da Silva ${ }^{3}$
}

\begin{abstract}
Objetivo: identificar potencialidades e desafios do trabalho de uma Equipe Itinerante de Saúde Mental no cuidado aos usuários de drogas. Método: estudo qualitativo realizado com uma equipe itinerante de um município do Rio Grande do Sul, Brasil. Os dados foram coletados por meio da observação participante e entrevista semiestruturada com os profissionais no ano de 2015. Para a análise dos dados, utilizou-se a Análise de Conteúdo Temática. Resultados: entre as potencialidades, destaca-se a própria criação deste serviço para atuar com o setor judiciário. Com relação aos desafios, elencou-se a dificuldade de discutir sobre a internação com a família. Conclusão: as inovações e os desafios são inerentes ao modo psicossocial no qual as situações complexas envolvem o território e a organização dos serviços de saúde.

Descritores: Saúde Mental; Reforma dos Serviços de Saúde; Políticas Públicas; Usuários de Drogas.
\end{abstract}

\footnotetext{
* Artigo extraído da dissertação de mestrado "O cuidado ao usuário de drogas: uma análise das tecnologias presentes no cotidiano do trabalho de uma equipe itinerante", apresentada à Escola de Enfermagem, Universidade Federal do Rio Grande do Sul, Porto Alegre, RS, Brasil.

${ }_{1}$ Universidade Federal do Rio Grande do Sul, Escola de Enfermagem, Porto Alegre, RS, Brasil.

${ }^{2}$ Secretaria Municípal de Saúde, Atenção Básica, Capão do Leão, RS, Brasil.

${ }^{3}$ Universidade Federal do Pampa, Uruguaiana, RS, Brasil.
} 


\section{Potentials and challenges of the itinerant team's work on drug user care}

Objective: to identify potentialities and challenges of the work of an itinerant mental health team in the care of drug users. Method: a qualitative study with an itinerant team, from a city in Rio Grande do Sul, Brazil. Data was collected through participant observation and a semistructured interview with professionals in the year 2015. For the data analysis, the Thematic Content Analysis was used. Results: among the potentialities, the very creation of this service to act with the judicial sector stands out. With regard to the challenges, the difficulty of discussing hospitalization with the family was highlighted. Conclusion: innovations and challenges are inherent in the psychosocial way in which complex situations involve the territory and the organization of health services.

Descriptors: Mental Health; Health Care Reform; Public Policies; Drug Users.

\section{Potencialidades y límites del trabajo de un equipo itinerante en cuidado a consumidores de drogas}

Objetivo: identificar potencialidades y limites del trabajo de un equipo itinerante em salud mental para el cuidado a consumidores de drogas. Método: estudio cualitativo realizado e nun equipo itinerante de una ciudad del Estado de Rio Grande do Sul, Brasil. Los datos fueran recolectados a partir de observación participante y entrevista semestructurada com los profesionales en 2015. Para el análisis de los datos se utilizo el Análisis de Contenido Temático. Resultados: entre las potencialidades, se destacaron la propia creación del servicio para actuar com la justicia. Sobre los desafíos se destacaron la internación psiquiátrica. Conclusão: las innovaciones y los desafios son típicos del modo psicosocial, em lo que lãs situaciones complejas envuelven el territorio y la organización de los servicios del salud.

Descriptores: Salud Mental; Reforma de la Atención de Salud; Políticas Públicas; Consumidores de Drogas.

\section{Introdução}

$\mathrm{Na}$ atenção aos usuários de drogas, as equipes itinerantes têm sido consideradas potentes no cuidado aos indivíduos e famílias. A itinerância é um modo de operacionalizar o cuidado no território que prevê o deslocamento dos profissionais para os espaços de moradia do usuário, com a produção de um cuidado contextualizado à existência humana, e pretende garantir a ruptura com lógicas hegemônicas normativas e reducionistas ${ }^{(1)}$.
As equipes itinerantes assumem um papel importante na atenção aos usuários de drogas, moradores de rua e outros grupos vulneráveis que não se adaptam a protocolos clínicos instituídos nos serviços. Essas equipes oportunizam a garantia do direito do encontro nos territórios com a identificação de suas necessidades sociais e de saúde, escuta, diálogo e participação na construção de estratégias de cuidado em rede ${ }^{(1-2)}$.

A criação de equipes itinerantes é fruto do processo de Reforma Psiquiátrica em curso no país. 
Seus avanços promoveram a construção de novas práticas de cuidado no campo da saúde mental que se materializam junto aos espaços do cotidiano dos usuários, o território, no qual o sofrimento psíquico é tomado como objeto complexo de vida, de múltiplas dimensões, e cuja perspectiva de cuidado leva em consideração os diversos modos de viver ${ }^{(3)}$.

Nessa perspectiva, os profissionais têm maior liberdade para investir e inventar diferentes modos de cuidar podendo integrar e articular ações territoriais e intersetoriais tais como vêm sendo desenvolvidas pelas equipes itinerantes. Assim, no ano de 2014, foi instituída oficialmente a primeira Equipe Itinerante de Saúde Mental de um município da região metropolitana de Porto Alegre, Rio Grande do Sul (RS). A formação dessa equipe aproxima dois grandes setores - o setor saúde e o setor judiciário.

Ajudicialização da saúde mental ganhou evidência nos últimos anos pelo aumento do uso abusivo de drogas e com as internações compulsórias usadas, muitas vezes, como uma estratégia de tratamento destinada a responder a uma demanda crítica - "apagar incêndio". A internação compulsória está prevista na Lei 10.2016/2001 (lei da reforma psiquiátrica brasileira) sob a deliberação da Justiça, que é responsável, também, por avaliar as condições de segurança do estabelecimento de saúde quanto à salvaguarda do usuário, dos demais internados e funcionários da instituição(4).

A formação e a criação da Equipe Itinerante de Saúde Mental são uma proposta inovadora que tem como objetivos diminuir a judicialização da saúde mental no município e qualificar a oferta de cuidado ao usuário de drogas e sua família. Ao receber os processos judiciais, os profissionais avaliam cada caso e realizam os primeiros encaminhamentos para inserir o usuário na rede de cuidado, respondem oficialmente ao setor judiciário em relação às condutas tomadas e, além disso, cabe a essa equipe fortalecer, com o setor judiciário e demais espaços sociais, questões emblemáticas relacionadas ao cuidado do usuário de drogas.

Diante da complexidade do cuidado que essas equipes enfrentam ao buscar construir uma interface com o território e inovar o modus operandi do trabalho em saúde é que este estudo tem por objetivo: identificar potencialidades e desafios do trabalho de uma Equipe Itinerante de Saúde Mental no cuidado aos usuários de drogas. Os resultados deste estudo podem colaborar para a problematização e a transformação de realidades semelhantes ao deste estudo na equalização dos processos de judicialização do cuidado ao usuário de drogas.

\section{Método}

Trata-se de um estudo de abordagem qualitativa orientado pelo referencial teórico do processo de trabalho em saúde. A pesquisa qualitativa utiliza-se do universo dos significados, aspirações, crenças, valores, atitudes e cultura, pois entende-se que esse conjunto de fenômenos humanos é parte da realidade social(5).

E o processo de trabalho na saúde ocorre em ato, na realização da ação, cujo produto é inseparável do processo de sua formação. O processo de trabalho é realizado por meio de tecnologias, os saberes operantes que constituem o instrumental teórico-conceitual que embasa a ação em saúde para a produção de práticas voltadas para o objeto, indivíduo ou grupos sadios/ doentes com diferentes necessidades ${ }^{(6)}$.

O campo de estudo foi a rede de saúde mental de um município da região metropolitana de Porto Alegre do Estado do Rio Grande do Sul, Brasil, área de atuação da Equipe Itinerante de Saúde Mental. Os participantes do estudo foram os profissionais que compõem essa equipe, três psicólogas e uma oficineira, entretanto, conforme os critérios de exclusão para participar da pesquisa - estar afastado ou de férias durante o período da coleta de dados -, a oficineira não participou na fase de coleta das entrevistas.

A coleta de dados foi realizada por meio de entrevista semiestruturada e da observação participante. A observação participante e a análise documental foram realizadas de julho a setembro de 2015 , totalizando 180 horas. Na observação participante, foi acompanhado o processo de trabalho da equipe em visitas domiciliares, no acompanhamento de usuários e famílias, em reuniões com serviços de saúde mental e em outros dispositivos da rede de cuidado, bem como na elaboração de parecer técnico, ao Ministério Público, referente à demanda de avaliação da necessidade de internação compulsória.

Para realizar a análise dos dados obtidos no estudo, utilizou-se a Análise de Conteúdo Temática, ${ }^{5}$ que é composta por três etapas: Pré-Análise, Exploração do Material e Tratamento dos Resultados Inferência/ Interpretação. Na primeira etapa, foi realizada a leitura flutuante e exaustiva de todo o material. Na segunda fase, foi realizada a análise dos dados, separando do texto fragmentos e trechos que foram distribuídos em tópicos, identificados como unidade de informação e, em seguida, foram aproximadas as unidades de informações semelhantes dando origem às unidades de sentido que, a partir da aproximação e do trabalho analítico das unidades de sentido, culminaram nas categorias analíticas. Por fim, na terceira etapa, foi realizada uma síntese interpretativa a partir do 
tratamento dos resultados obtidos, sendo estes submetidos a operações complexas ou simples que permitiram ressaltar as informações obtidas ${ }^{(5)}$.

Conforme a Resolução n. ${ }^{\circ} 466 / 2012$, do Conselho Nacional de Saúde, o estudo contemplou as prerrogativas bioéticas e obteve-se a aprovação do Comitê de Ética em Pesquisa, em julho de 2015, por meio do número de parecer: 1.144.089. Os participantes do estudo assinaram o Termo de Consentimento Livre e Informado e foram identificados com a letra $\mathrm{T}$ de trabalhador $\mathrm{e} o$ número correspondente à entrevista, como T01, T02, respeitando-se o anonimato. Os trechos do diário de campo foram representados pela sigla DC.

\section{Resultados e discussão}

\section{Potencialidades do trabalho da Equipe Itinerante de Saúde Mental}

As equipes itinerantes, por atuar na rua e nos locais de produção de vida, tornam-se importantes ferramentas tecnológicas de cuidado identificando situações de conflito, de rompimento familiar, de abandono escolar, de violência, entre outras situações vulneráveis que necessitam do olhar de profissionais dispostos a fazer o seu papel em saúde.

A autonomia de criar alternativas que possam responder a demandas locais de saúde, tal como a criação da equipe itinerante, é uma das características dos modos de organização do sistema de saúde brasileiro. Essa autonomia dá condições, ao município, de pensar nas suas reais necessidades, de criar serviços ou estratégias de cuidado, assumindo as responsabilidades pela tomada de decisão local.

Nesse caso, os trabalhadores apontam que a própria criação da equipe foi uma das potencialidades não somente pelo fato de que existem muitas demandas judiciais em saúde mental, mas também por ser a Equipe Itinerante uma estratégia que pode promover o cuidado nesse campo: Eu acho que a itinerante é uma inovação assim, sabe. Eu acho que é uma inovação no cuidado, eu acho que ela tem muito mais chances de ser um cuidado resolutivo do que cumprir simplesmente uma determinação judicial [...]. Eu acho que tu podendo pensar e sinalizar e construir mesmo que seja um caso que precise, de fato, de uma internação, que isso seja colocado de um jeito diferente para o usuário; vai ser muito mais terapêutico do que tu cumprir, na íntegra, o que está ali no preto e no branco (T01). Eu acho que é mais uma coisa que foi criada pensando no cuidado e no cuidado em liberdade do usuário [...] uma das coisas que venho, junto com isso, da criação da itinerante. Eu acho que foi isso da saúde mental bancar, junto ao jurídico, que não quem tem propriedade se aquela pessoa precisa ser internada ou não é a saúde mental não é o juiz (T03).

A mudança de modelo redireciona o cuidado para o território de vida das pessoas. A proposta de mudança do modelo assistencial em saúde é expressa na VIII Conferência Nacional de Saúde, em 1986, e consagrada no cenário brasileiro com a criação do Sistema Único de Saúde, que trouxe princípios como a universalidade, a equidade e a integralidade, as diretrizes que devem reorientar o processo de cuidado em saúde e a ampliação do conceito de saúde como resultado das condições sociais de vida ${ }^{(3)}$. São por essas mudanças de modelo que o município em estudo consegue inovar suas práticas construindo novas formas de atuar frente às necessidades e demandas do território.

Nesse sentido, pensando no contexto "macropolítico" do processo, o município vem propondo novos modos de cuidado por meio da implantação de equipes itinerantes de saúde. A criação da Equipe Itinerante de Saúde Mental, nessa perspectiva, é uma inovação porque a judicialização da saúde é um processo corriqueiro no local estudado. Já no contexto "micropolítico", ou seja, no local de funcionamento cotidiano da rede e da própria equipe nessa rede, há outros elementos envolvidos que facilitam a atuação dos profissionais. T02, a seguir, destaca a autonomia de trabalhar nessa equipe, além da disponibilidade de um carro, algo não recorrente em outras realidades assistenciais: A gente tem bastante, não sei se bastante, mas bem mais que outros serviços é a nossa possibilidade de autonomia do trabalho ali, de desenhar [...] A gente tem um transporte disponível que facilita muito, não era pra ser, mas a gente sabe que tem muitos colegas que trabalham sem transporte [...]. É uma potencialidade de condição de trabalho (T02).

T02 destaca a autonomia, enquanto potencialidade do trabalho da equipe, para construir redes externas aos serviços. No entanto, compreende-se que é preciso relativizar essa autonomia do trabalhador, pois suas ações são condicionadas pela organização técnico-organizacional, pelas influências sociopolíticas presentes nos espaços de trabalho e pela produção de cuidado, que é caracterizada por uma dada formação ${ }^{(7)}$.

A equipe apresenta mais condições de reinventar o trabalho, ou seja, de pensar e organizar os processos que o envolvem desenhando alternativas de cuidado. E esse maior grau de autonomia se fortalece quando o trabalhador encontra, no serviço, os próprios meios para favorecer a realização de suas atividades, como ter um veículo à disposição.

Estudos apontam importantes dificuldades na articulação entre os serviços especializados e a rede básica no cuidado em saúde mental( ${ }^{(8-9)}$. Assim, o 
automóvel pode colaborar para a organização dos processos de trabalho centrados na necessidade de conhecer os territórios de moradia dos usuários e familiares, na articulação com os serviços da rede, nas redes formais e informais de cuidado, os seus espaços comuns de socialização e dar celeridade à resposta das demandas e ações da equipe.

A equipe observa, ainda, avanços na construção de novos projetos de cuidado e diálogo com a atenção básica, mesmo que ela ainda tenha dificuldades de compreender o seu papel em relação ao cuidado na saúde mental: Na própria atenção básica, em termos de saúde mental, como um todo, eu acho que tem se conseguido trabalhar ainda isso de uma maneira bem bacana, assim, com a atenção básica através do matriciamento [...] mas, assim, tu vê uma dificuldade muito grande das pessoas, assim, aceitarem que a atenção básica também tem que dar conta do cuidado de saúde mental em território (T03).

Em um estudo realizado com profissionais da Estratégia Saúde da Família, identificou-se a interação entre os campos saúde mental e atenção primária em saúde (APS) por meio do envolvimento de especialistas e generalistas, pelo matriciamento, proporcionando novas relações e interações profissionais. Isso promove um novo modelo de saúde pública que implica práticas de cuidado na perspectiva da integralidade ${ }^{(10)}$. Nesse sentido, é preciso investir na formação profissional e nas práticas de matriciamento em saúde mental(8).

Outra potencialidade relacionada ao trabalho da Equipe Itinerante foi o avanço das ações conjuntas com o Centro de Atenção Psicossocial Álcool e outras drogas (CAPS AD). Entre essas ações, destacam-se a nomeação de um técnico de referência do serviço especializado para receber as demandas da Equipe Itinerante e o aumento da frequência de reuniões entre as equipes, como exposto a seguir: Eu acho que a relação com o CAPS AD ela tem melhorado bastante [...] A gente, com eles, conseguiu estipular algumas coisas do tipo técnico de referência da equipe que possam ficar mais atrelados, assim, mais responsáveis por esse cuidado com a itinerante, a gente tem conseguido fazer reuniões, agora, a gente tem conseguido fazer mais em seguida (T03). Às 9 horas, uma técnica do CAPS AD veio convidar uma das trabalhadoras da Equipe Itinerante para participar de uma reunião com o CREAS [Centro de Referência Especializado em Assistência Social] para discutir um caso, que é um processo judicial. T03 questiona o motivo da reunião e a colega do CAPS AD diz que o CREAS acompanha a família (mãe e filho) e que a mãe é idosa e o filho não está conseguindo se organizar para cuidar dela (DC).

Embora o CAPS AD seja um potente local de cuidado a usuários de drogas, também é evidente a necessidade de expandir as ações para fora dos serviços no sentido de integrar melhor a rede de atenção psicossocial e os trabalhadores envolvidos. Os trabalhadores do CAPS AD sentem-se restritos aos espaços intramuros quando deveriam, segundo as premissas do modelo de Atenção Psicossocial, circular mais pelo território(11). Reuniões para discutir casos podem fazer parte das estratégias que possibilitam a interação entre os serviços ampliando a problematização de situações complexas e o planejamento de ações articuladas entre diferentes serviços e interlocutores na atenção aos usuários dos serviços.

Assim, o ato de cuidar de usuários de drogas, em serviços comunitários, exige, dos profissionais, um conhecimento reflexivo e criativo, pois é preciso aprender com o imediatismo do usuário, ao tempo em que se tenta ressignificar a relação estabelecida com o uso de uma determinada droga ${ }^{(12)}$.

Nesse caso, os serviços de saúde devem se organizar de modo a atender às necessidades do usuário envolvendo não apenas o conhecimento técnico sobre a substância, mas, também, a realidade vivida pelo sujeito, seu modo de vida, seu espaço e suas relações.

\section{Desafios do trabalho da Equipe Itinerante}

A característica preservada da Equipe Itinerante, de ser um serviço com atuação mais voltada para o território, permite que ela se organize para compreender que a vida, lá fora, possui nuances bem mais singulares e que os serviços de saúde nem sempre estão acostumados a trabalhar. Uma vez que o sujeito vive em um território vivo, isto é, circula por ele, organiza seus projetos e suas relações. Assim, é possível perceber que as equipes encontram dificuldades situadas nesse campo das relações.

T02 destaca que um dos desafios da equipe é a dificuldade para encontrar a pessoa na residência: Acho que um desafio grande que é, por vezes, encontrar o usuário; a gente recebe o processo e tem que se organizar e dentro do nosso horário de trabalho, horário que tem carro e tal, às vezes, com outros serviços junto é toda uma articulação pra ir até a residência e, às vezes, esse usuário não está [...] (T02). Foram para o endereço fornecido pela avó do jovem uma profissional da equipe itinerante e um profissional do CAPS Infantojuvenil, mas não encontram a casa, desceram do carro e caminharam pela rua, perguntando para os moradores, mas ninguém conhecia o menino e nem a sua mãe (DC).

Por essa natureza "extramuros", que confere contornos interessantes relacionados à autonomia do fazer, a equipe também se depara com as peculiaridades 
existentes em cada território em que atua. Nesse caso, o que, para elas, corresponde a uma dificuldade, o fato de não encontrar o usuário em casa, se entende que isso esteja relacionado ao funcionamento das pessoas no seu espaço de vida no qual elas organizam os seus horários e estabelecem as suas prioridades. Essa característica é um grande desafio para o trabalho em saúde mental, pois trata-se do reconhecimento desse território como algo "dinâmico" e que foge às linhas assépticas dos serviços e seus modos de organização institucionalizados ${ }^{(13)}$

Assim, em qualquer visita domiciliar, é possível não encontrar a pessoa na residência, sendo necessário reinventar outros modos de encontro como, por exemplo, em uma quadra de futebol, na escola, na casa de um amigo, enfim, fazer itinerância é constituir maior trânsito nesses locais de circulação dos usuários.

No entanto, quando se fala sobre usuário de drogas, incorporam-se outros elementos nesse processo. Alguns estudos já vêm discutindo que o funcionamento do usuário de drogas e de suas famílias é afetado por conta de situações como a agressividade, a violência, a questão do tráfico e o rechaço social do usuário, colocando-o à margem da sociedade ${ }^{(11,14-16)}$. Outra dificuldade é a questão da violência da agressividade que, às vezes, é difícil de manejar com a familia, a questão de território dele que envolve tráfico [...] A polícia, que faz uma atuação supercomplicada e, daí, gera medo, gera raiva e revolta e daí, às vezes, até por desconhecimentos, os usuários relacionam muito polícia com poder público, então, fica um rechaço em relação a qualquer serviço [...] e eles sofrem violência mesmo, são muito mais marginalizados, são excluídos, passa por todas as questões de moralismo [...].(T02).

Em estudos com usuários de crack demonstrou-se que as relações familiares são frágeis e permeadas de conflitos, uma vez que a droga atua, principalmente, na ruptura de laços afetivos entre os membros da família. O uso de drogas como o crack gera níveis mais altos de conflitos do que outras drogas, o que pode estar ligado às alterações que a substância provoca no organismo da pessoa ${ }^{(11,14)}$.

O modo de cuidado proposto pelas políticas públicas é o que possibilita esse encontro entre os profissionais da saúde e as famílias, em seu contexto de vida, revelando a complexidade das relações, as histórias de vida e o sofrimento mental do usuário e da família. Esse novo modo de atuar demanda novas formas de cuidar, de lidar com os imprevistos, de recriar frente à necessidade de cada pessoa e, por isso, é desafiador e exige mais dos trabalhadores da saúde mental.

Outro desafio enfrentado pelos usuários de drogas é a relação deste com a sociedade. O usuário de drogas é, constantemente, afetado pelo processo de estigmatização que gera restrição social, baixa autoestima e perspectivas limitadas de recuperação que interferem, de modo negativo, no tratamento. A ação da mídia, que transmite informações deturpadas, somada à falta de esclarecimento sobre o sofrimento psíquico, gera uma percepção do usuário de drogas como incapaz de se recuperar e muitos são vistos como temidos. Desse modo, eles sofrem com o preconceito e a criminalização, com a desconfiança e os estereótipos ${ }^{(17)}$.

Nessa mesma vertente, destaca-se que a mídia, por meio da televisão, principalmente, relaciona modos simbólicos sobre o uso de drogas lançando um efeito de individualização que produz e/ou mantém um imaginário social do usuário de drogas como doente ou delinquente. Em ambos os casos, o destino do usuário de drogas é a punição ora por meio da internação hospitalar, ora por meio do aprisionamento ${ }^{(18)}$.

Assim, sair das paredes do CAPS e ir ao encontro do desconhecido representam uma valiosa ferramenta de cuidado em saúde mental que possibilita novas formas de atenção aos usuários de drogas. Os conflitos familiares vividos em função do uso de drogas fazem com que o trabalhador se depare com um dos motivos que os levam a procurar a internação como refúgio. $O$ fragmento de T03 destaca essa questão: [...] a família solicita muito, assim, a internação direto; acho que tem, de um modo geral, na sociedade, assim, uma fantasia de que tu internando a pessoa, tu vai tirar aquilo ali com a mão [...] claro que, muitas vezes, a família já está cansada e já não aguenta mais muitas vezes [...] Muitas vezes acontece, de fato, de ter um risco iminente pra família e ai é isso que tu tem que poder avaliar [...] tu poder colocar pra aquela família que, talvez, a expectativa que ela tenha não vai ser exatamente atendida, que isso também faz parte do cuidado da saúde mental [...] (T03).

A equipe possui o desafio de desconstruir, com a família, o imaginário social de que a internação irá resolver as demandas em saúde do seu familiar. E, para além disso, construir, com a família, os meios de cuidado de si e do usuário, pois há uma sobrecarga familiar que também precisa ser atendida pelas equipes de saúde.

A preocupação com o uso da internação é devido ao fato histórico de ter sido utilizada como ferramenta de isolamento social, maus-tratos e punição. Ou seja, a internação foi usada secularmente como a principal opção, no entanto, era um dispositivo de isolamento, não havendo outros modos de cuidado em liberdade. Atualmente, a internação é uma tecnologia de cuidado, entre outras modalidades terapêuticas, que deve ser utilizada quando houver a necessidade dessa intervenção, garantindo o acesso aos leitos psiquiátricos em hospitais gerais e o acompanhamento do caso pelas equipes do território. 
Estudo de avaliação da inserção da pessoa com deficiência intelectual na Rede de Atenção Psicossocial identificou itinerários marcados pela institucionalização com importante barreira de acesso nos CAPS e o uso da internação como primeira opção(9). Dessa maneira, é necessário reavaliar o modo como as internações estão sendo usadas e propor métodos que valorizem o uso de serviços substitutivos de saúde mental como o acompanhamento dos jovens em CAPS e em outros serviços da rede de cuidado.

Outro desafio do trabalho da Equipe Itinerante está na ressignificação do olhar do trabalhador que, muitas vezes, perpetua o olhar moralizante e excludente da sociedade a qual pertence. [...] a gente consegue está marcando reuniões com a rede ampliada, assim, entrando em contato com os outros serviços, com os outros dispositivos, mas é sempre muito difícil assim também, principalmente, quando envolve o uso de drogas e muito talvez também por essa, por esse imaginário social em relação ao uso de drogas, sabe, e mesmo com profissionais e colegas de outros setores tu encontra um certo preconceito (T03).

Nesta lógica, os profissionais que compõem os serviços de cuidado também são influenciados pelo moralismo existente na sociedade. A Equipe Itinerante deve continuar tecendo as redes de cuidado e, nestas, devem promover diálogos que desconstruam imaginários sociais depreciativos e estigmatizantes em relação ao usuário de drogas.

É necessário que os profissionais da saúde identifiquem os próprios preconceitos em relação ao consumo de drogas e todas as questões existentes nesse fenômeno de uso. Atitude não reflexiva e um olhar preconceituoso geram grandes sofrimentos, principalmente, ao usuário, que já enfrenta os problemas físicos, psicológicos e sociais relacionados ao consumo de drogas ${ }^{(16)}$.

O município pode investir em programas de educação permanente, que incluam o tema "drogas" em suas agendas, com vistas à desconstrução de estigmas, à diminuição de preconceitos e à reorientação dos serviços para atender aos problemas complexos gerados pelo abuso de drogas. Pode-se ajudar na maior aproximação de famílias e usuários, dos serviços e profissionais reconhecendo-os como parceiros e corresponsáveis pelo tratamento.

Desse modo, as inovações que permeiam o trabalho da Equipe Itinerante também convivem, cotidianamente, com os desafios impostos por um modo de funcionar mais próximo das pessoas do que dos serviços. Essa dialética de cuidar, que não é regular, é típica do modo psicossocial como possibilidade capaz de trazer originalidade e mais diversidade à prática em saúde mental, apesar de trazer, também, novos desafios para o processo.

\section{Conclusão}

Em relação às potencialidades do trabalho da Equipe Itinerante de Saúde Mental, destaca-se a criação do próprio serviço para atuar com o setor judiciário e para diminuir a judicialização da saúde. Além disso, há a autonomia para realizar as atividades, desenhando novos formas de cuidar, e o fato de ter um veículo para favorecer e agilizar o cumprimento de tarefas do serviço. Outras potencialidades do serviço foram os avanços na construção de pontes de diálogos e cuidado compartilhados com o CAPS AD e a atenção básica. Portanto, as potencialidades da equipe implicam, além de sua proposta mais resolutiva e terapêutica, a forma com que os trabalhadores se organizam para tal reafirmando-se a inovação e a possibilidade de um fazer diferente proporcionado pelo cuidado itinerante no território.

No que se refere aos desafios da Equipe Itinerante de Saúde Mental, os participantes trouxeram as situações peculiares do trabalho no território, como não encontrar o usuário em sua residência, o manejo dos conflitos entre o usuário e a família e as questões de violência, tráfico e o preconceito da sociedade com o usuário de drogas. Além desses entraves, é um desafio desconstruir, com a família, que a internação é apenas uma das modalidades terapêuticas de um cardápio amplo de opções que podem responder, ou não, às necessidades dos usuários e ao trabalho em rede.

Por fim, espera-se que o estudo possibilite novas reflexões sobre o cuidado de usuários de drogas e a judicialização da saúde mental. E que as potencialidades e os desafios dessa equipe itinerante, enquanto processos inovadores na área da saúde, possam contribuir para a criação de novos dispositivos de cuidado.

\section{Referências}

1. Lemke RA, Silva RAN da. Um estudo sobre a itinerância como estratégia de cuidado no contexto das políticas públicas de saúde no Brasil. Physis. [Internet]. 2011 [Acesso 29 jan 2016]; 21(3): 979-1004. Disponível em: http:// www.scielo.br/scielo.php?script=sci_arttext\&pid=S010373312011000300012\&Ing=en. http://dx.doi.org/10.1590/ S0103-73312011000300012.

2. Pires LB. A humanização no cuidado de pessoas em situação de vulnerabilidade: a experiência vivenciada no consultório na rua de Campinas/SP. [Internet] São Paulo 
(SP): Universidade Estadual de Campinas; 2014 [Acesso 16 abril 2016]; Disponível em: https://www.fcm.unicamp.br/ $\mathrm{fcm} /$ sites/default/files/paganex/tcc2013liviabuenopires.pdf 3. Yasui S, Luzio CA, Amarante P. Atenção psicossocial e atenção básica: a vida como ela é no território. Rev Polis Psique. [Internet]. 2018 [Acesso 27 ago 2018]; 8(1): 173-90. Disponível em: http://seer.ufrgs.br/PolisePsique/ article/view/80426/pdf

4. Lei 10.216 (BR). Dispõe sobre a proteção e os direitos das pessoas portadoras de transtornos mentais que redireciona o modelo assistencial em saúde mental de 2001. Brasília, 06 de abril de 2001.

5. Minayo, MCS. O desafio do conhecimento: pesquisa qualitativa em saúde. 14a ed. São Paulo: Hucitec; 2014.

6. Gonçalves RBM. Tecnologias e organização social das práticas de saúde: características tecnológicas de processo de trabalho na rede estadual de centros de saúde de São Paulo. São Paulo: Hucitec; 1994.

7. Fischborn AF, Cadoná MA. Trabalho e autonomia dos trabalhadores em saúde: considerações sobre pressupostos teórico e metodológicos de análise do trabalho em saúde. Saúde Soc. [Internet]. 2018 [Acesso 27 ago 2018]; 27(1):227-37. Disponível em: http://www. scielo.br/scielo.php?pid=S010412902018000100227\&sc ript=sci_abstract\&tlng=pt

8. Pinho LB de, Siniak DS. The role of primary care in the assistance to crack user: opinion from users, collaborators and managers of the system. SMAD, Rev Eletrônica Saúde Mental Álcool Drog. [Internet]. 2017 [cited Ago 28 2018]; 13(1): 30-6. Available from: http://pepsic. bvsalud.org/scielo.php?script=sci_arttext\&pid=S180669762017000100005\&lng=pt\&nrm=iso

9. Hirdes A. A perspectiva dos profissionais da Atenção Primária à Saúde sobre o apoio matricial em saúde mental. Ciênc Saúde Coletiva. [Internet]. 2015 Feb [Acesso 29 ago 2018]; 20(2): 371-82. Disponível em: http://www. scielo.br/scielo.php?script=sci_arttext $\&$ pid=S1413$81232015000200371 \&$ Ing=en. http://dx.doi. org/10.1590/1413-81232015202.11122014.

10. Pinho LB de. Avaliação qualitativa da rede de serviços de saúde mental de Viamão para atendimento a usuários de crack. Relatório de Pesquisa. Relatório final. Edital do Conselho Nacional de Desenvolvimento Científico e Tecnológico $N^{\circ} 41 / 2010$. Universidade Federal do Rio Grande do Sul; 2014.

11. Lacchini AJB, Nasi C, Oliveira GC, Barbosa LB de, Schneider JF. Características de usuários de crack atendidos em um Centro de Atenção Psicossocial: concepção da equipe. Rev Eletr Enferm. [Internet]. 2015 [Acesso 10 fev 2016]; 17(2): 196-204. Disponível em: https://www.fen.ufg.br/revista/v17/n2/pdf/v17n2a04.pdf 12. Silva AB, Pinho LB de. Território e saúde mental: contribuições conceituais da geografia para o campo psicossocial. Rev Enferm UERJ. [Internet]. 2015 [Acesso 10 jan 2016]; 23(3): 420-4. Disponível em: http://www. epublicacoes.uerj.br/index.php/enfermagemuerj/article/ view/10091

13. Golub A, Dunlap E, Benoit E. Drug Use and Conflict in Inner-City African-American Relationships in the 2000s†. J Psychoactive Drugs. [Internet]. 2010 [cited Jan 10, 2016]; 42(3): 327-37. Available from: https://www.ncbi.nlm.nih. gov/pmc/articles/PMC3743426/

14. Garcia IP. A dependência química no contexto familiar: Uma análise do relato de três mães. Psicologia.pt [Internet] 2018 Apr [Acesso 28 ago 2018]; 1-14. Disponível em: http://www.psicologia.pt/artigos/textos/A1198.pdf

15. Souza WC, Alencar IM, Ribeiro PE. Cuidado integral à saúde de usuários de crack e outras drogas: ação de rede entre consultório de rua e atenção básica. Rev Contrib Ciências Soc. [Internet]. nov 2015 [Acesso 14 jan 2016]; (30): [cerca de 18 p.]. Disponível em: http://www.eumed. net/rev/cccss/2015/04/crack.html

16. Ronzani TM, Noto AR, Silveira PS. Reduzindo o estigma entre usuários de drogas guia para profissionais e gestores. Juiz de Fora: Editora UFJF; 2015

17. Romanini M, Roso A. Midiatização da Cultura, Criminalização e Patologização dos Usuários de Crack: Discursos e Políticas. Temas Psicol. [Internet]. 2013 [Acesso 20 jan 2016]; 21(2): 483-97. Disponível em: http://pepsic. bvsalud.org/scielo.php?script=sci_arttext\&pid=S1413389X2013000200014

18. Surjus LTLS, Campos RTO. Indicadores de avaliação da inserção de pessoas com deficiência intelectual na Rede de Atenção Psicossocial. Saúde Debate. [Internet]. 2017 [Acesso 27 ago 2018]; 41(n. especial): 60-70. Disponível em: http://www.scielo.br/scielo.php?pid=S010311042017 000500060\&script=sci_abstract\&tlng=pt 